\title{
TYPING ERRORS AS CLUES TO SERIAL ORDERING MECHANISMS IN LANGUAGE BEHAVIOUR*
}

\author{
Peter F. Macneilage

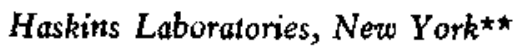

\begin{abstract}
623 typing errors produced by 5 subjects in a free typing situation were classified into two main categories : (a) spatial errors, in which a letter adjacent on the keyboard to the correct letter was typed, and (b) temporal erross, or errors in the order in which the required letters were typed. Spatial errors were mostly associated with the left hand and keyboard difficulty, while temporal errors mostly arose from language structure variables. Results were incompatible with "one stage" or "chaining" theories of serial ordering. They suggested a hierarchical or "three stage" outpur mechanism consssting of (a) a "determining tendency", (b) a programming mechanism, reflected by temporal errors, and (c) a relatively separate executive mechanisin, reflected by spatial errors. Some characteristics of the programming mechanism are discussed.
\end{abstract}

What mechanisms control the sequential output of language? Lashley (1951) in calling attention to the problem of serial ordering of behaviour in general, dismisses as inadequate the only theory that had attempted to deal with this problem, the stimulus-tesponse or "chaining" theory. This theory maintains that "chains" of behaviour result from a response itself providing a stimulus (largely proprioceptive) which directly produces the next response. On the other hand, Lasbley argues that there must be a hierarchy of determinants for any given sequence of behaviour, and makes some attempt to specify what the determinants might be. In doing this he relies rather heavily on evidence from mistakes of language sequencing such as spoonerisms and typing errors. Although cogent, and intuitively impressive, this evidence comes largely from incidental observations of Lashley's own typing and speaking, and that of others. This material is, of necessity, selectively and unsystematically compiled. Of the two language producing processes, typing and speech, typing has the methodological advantage of occurring in discrete response units that are automatically recorded. But in an ordinary typing situation, many kinds of error are possible, and a number of variables could be influential in any type of error. This possibility of multivariate determination of errors could serve to make analysis of typing behaviour in a free situation a difficult task. Nevertheless, in lieu of a number of experiments reducing the number of variables (rows, columns, fingers, letters, hands, letter and digram frequencies, etc.) and controlling the rôle of each,

* The investigation was supported in part by PHS Research Grant DE 01774 from the National Instittule of Dental Research, National Institutes of Health, Public Health Service, U.S. Department of Health, Educarion and Welfare. The author is grateful to Michael Studdert-Kennedy who gave much advice on statistical and other problems. 


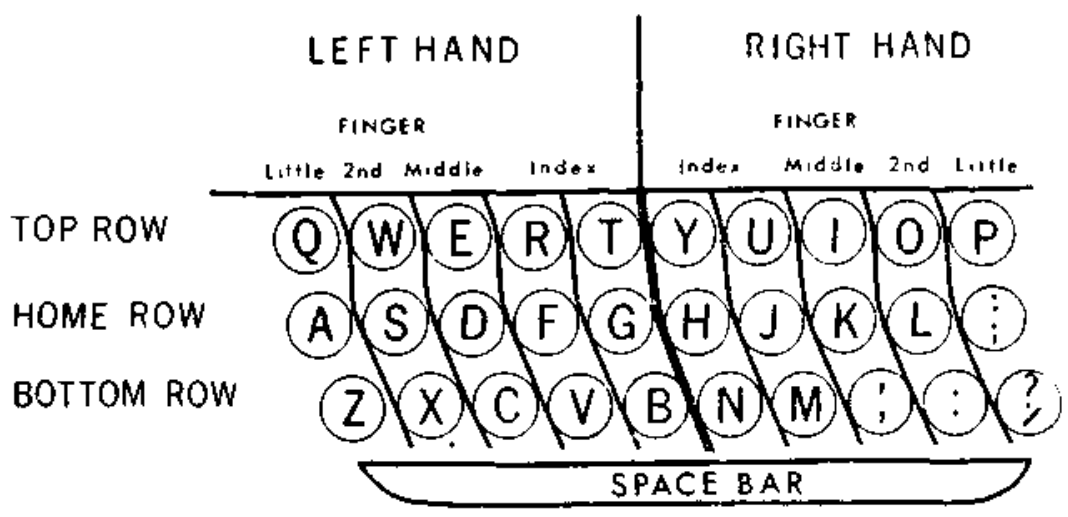

Fig. 1. Sketch of the typewriter keyboard showing the hand and finger responsible for each column.

more information on the serial ordering process than Lashley has presented may be gained from a systematic collection and analysis of a corpus of errors occurring in ordinary typing situations. The present paper is a report of such an analysis and a consideration of the results in relation to the theories of serial ordering mentioned above.

\section{METHOD}

Subjects were 5 female college sophomores each of whom used the same orthodox system of touch typing. Subjects' typing speeds ranged from 30-45 words per minute where a word was defined as 6 letters with "space" counting as a letter. No penalty was paid for errors in this calculation. These speeds are roughly equivalent to that of an average professional typist. Three of the subjects were right banded, and 2 wrote left banded but performed some other babitual functions with their right hands.

The material from which the typing errors were collected was a number of experimental reports of work done in a laboratory course in experimental psychology. Subjects typed the reports at home from rough written drafts. They were instructed to stop typing whenever they became aware that they had made an error, and to begin again at the beginning of the word in which the error had been made. This instruction beld only for continuous sequences involving the 26 lower case letters, and spaces. Capital letters, numerals, punctuation marks, and other symbols available on the keyboard were not considered. Neither was any written material associated 
with figures or tables. Fig. 1 is a sketch of the typewriter keyboard for reference during the description of error types that follows.

\section{Classification of Errors}

Spatial errors. Spatial errors result from typing a letter immediately adjacent on the keyboard to the one required by the copy. They were divided into :

(a) Horizontal errors. These consist of typing a letter immediately to the left or right of the correct letter, in the same row of the keyboard; e.g. "e" for " $t$ ", or " $d$ " for " $f$ ".

(b) Vertical errors. These result from typing a letter immediately above or below the correct letter in the same column of the keyboard ; e.g. " $f$ " for " $r$ ", or " $e$ " for " $d$ ".

(c) Diagonal errors. A letter is typed in $\mathbf{a}$ row and a column adjacent to that of the correct letter; e.g. " $d$ " for " $r$ ". (Note that this classification cannot show whether the error is made by the finger which should have made the correct response or not.)

Temporal errors. Temporal errors are errors in the order in which the required letters were typed. These were divided into :

(a) Reversal errors. Two letters next to each other in the correct sequence are reversed in their order ; e.g. "ht" for " th".

(b) Omission errors. One letter in a sequence is left out; e.g. "lenth" for "length".

(c) Equivocal errors. The letter one stroke ahead of the one required in the copy is typed, after which the subject stops (becoming aware of the error); e.g. "stiml--" for "stimulus". The error was named equivocal because it shows some conformity to types (a) and (b) but, since the subject stopped immediately after making it, cannot be categorized as either.

(d) Anticipation errors. A letter is typed which is required more than one stroke ahead of the place where it is mistakenly typed : e.g. " ext-" for " expected".

Miscellaneous errors. A number of other specific error types were distinguished :

(a) Interpolation. A letter apparently quite unrelated to the correct sequence is inserted in it ; e.g. "formend" for "formed".

(b) Phonemic errors. A letter pronounced similarly to that of the correct letter replaces the correct letter in the sequence ; e.g. "mac" for " makes".

(c) Type errors. One letter of a word is changed, making it into a word similar to the correct one but meaningless in context; e.g. "that "for "than".

(d) Contralateral errors. A stroke is typed using the same row and the corresponding finger to the correct one, but with the other hand; e.g. "treals" for "trials".

(e) Dynamics errors. The letter adjacent in the sequence to a letter which should have been typed twice, is typed twice instead; e.g. "eroors" for "errors". 
All other errors were placed into one of two remaining categories :

(a) Multiple classification errors. These are errors which can be placed in more than one category; e.g. "respression" for "repression", where the first " $s$ " could be an interpolation or an anticipation error.

(b) Unclassifiable errors. These are errors which could not be placed into any of the above categories; e.g. " condidioning" for " conditioning ".

Some general qualifications were placed upon the classification procedure. Nothing that could have been a simple spelling error was included in the sample. Except in the case of reversal etrors, every error involving more than one letter of the word was labelled "unclassifiable" because of the multiplicity of equally likely interpretations that they tended to encourage. The space gesture, made with the right thumb, was not considered to be part of the sequence. Therefore, it was not classed as a "letter" or a right hand gesture when analyses of hand sequence's and letter sequences adjacent to errors were being made. The only accasion where classification or analysis extended across the word boundary was in "anticipation" errors, which were, by definition, errors which spread across a larger piece of text than other errors. The error classification was made by the author. Another judge subsequently examined it, and any disagreements were resolved to the satisfaction of both parties.

\section{Results}

A summary of the frequencies of occurrence of the various types of error and of the percentage frequencies of the main classes of error is seen in Table 1. Approximately $70 \%$ of all errors was classifiable into particular single categories within the main classes of spatial, temporal and miscellaneous. Of the remainder, about $10 \%$ was not classifiable into any category, and about $20 \%$ was classifiable into more than one category.

Of the $\mathbf{4 4 2}$ classifiable errors, 387 fell into the two main classes of spatial and temporal errors, with slightly more spatial than temporal errors. The following analysis will, therefore, be mainly of spatial and temporal errors. Inspection of the data shows that the $S$ individual subjects each contributed to the various spatial and temporal ertor types in a manner which suggests that the error totals can be considered as group effects rather than effects produced largely by difficulties of individual typists.

Handedness. Table 2 shows the relative frequencies with which the left and right hands were involved in spatial and temporal errors. The result is best viewed in relation to an estimate of the relative frequencies with which strokes made with the left and right hand would normally occur. An estimate derived from Baddeley, Conrad and Thomson's (1960) letter frequency count of editorials from 5 issues of the London Times, shows the following percentages : Left hand- $55.5 \%$, Right band$44.5 \%$. This estimate is similar to others made for the same purpose (e.g. Dvorak, et $\alpha .$, 1936). Although the text of the students' reports obviously differs from this 


\section{TABle 1}

Relative frequencies of the various kinds of typing error.

SPATIAL, ERRORS

Horizontal

Vertical

Diagonal

Total

TEMPORAL ERRORS

Equivocal

Reversal

Omission

Anticipation

Total

Miscellaneous

Interpolation

Phonemic

Contralateral

Type

Dynamics

Total

MUL.TIPLE CATEGORY

UNCLASSIFLABLE

TOTAL f $\%$

148

50

10

208

88

32

26

33

179

11

2

11

24

7
55

112

69

623
33.4

28.7

$\%$
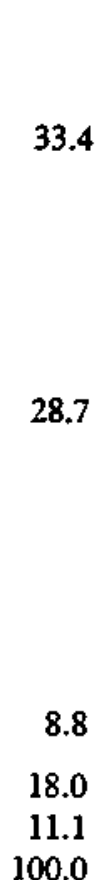
TABLE 2

Relative frequencies with which temporal and spatial errors were typed with left and right hands.

\begin{tabular}{|c|c|c|c|c|c|c|}
\hline \multirow{2}{*}{\multicolumn{7}{|c|}{ SPATIAL ERRORS }} \\
\hline & & & & & & \\
\hline Horizontal & 49 & 99 & \multicolumn{4}{|l|}{$<0.01$} \\
\hline Vertical & 8 & 42 & \multicolumn{4}{|l|}{$<0.01$} \\
\hline \multirow[t]{3}{*}{ Total } & 57 & 141 & & & & \\
\hline & \multicolumn{3}{|c|}{ "Advanced" Letter } & \multicolumn{3}{|c|}{ " Postponed" Letter } \\
\hline & Right & Left & & Right & Lef & \\
\hline \multicolumn{7}{|c|}{ TEMPORAL ERRORS } \\
\hline Equivocal & 42 & 46 & NS & 39 & 49 & NS \\
\hline Reversal & 15 & 17 & NS & 18 & 14 & NS \\
\hline Omission & 13 & 13 & NS & 13 & 13 & NS \\
\hline Anticipation & 12 & 21 & NS & 11 & 22 & NS \\
\hline Total & 82 & 97 & & 81 & 98 & \\
\hline
\end{tabular}

it was required in these error types. The postponed letter is taken to be the letter which should have occurred where the advanced letter occurred. Note that this letter is actually typed only in reversal errors. In summary, the left hand made significantly more spatial errors than did the right, with one exception, but there was no significant difference in temporal error frequency in the two hands.

Hond sequence. The data were analysed to see whether any particular sequences of left and right hand strokes were associated with any of the spatial or temporal error types. Expected frequencies for the 4 possible digram sequences (LL, LR, RL and RR) were derived from the Baddeley, Conrad and Thomson digram count and were used to evaluate the frequencies with which each of the types of spatial and temporal errors occurred either in first or last positions in the digrams. In spatial errors, the only result found was that any digram in which the left hand made the error occurred with significantly greater-than-chance frequency. This result therefore, is not an effect of band sequence itself, but merely one of handedness. No sequences involving iemporal errors occurred with frequencies beyond chance. Error totals were too small to justify trigram analysis.

Keyboard variables. The relation between observed and expected error percentages in columns of the keyboard is shown in Table 3. Expected percentages were again derived from Baddeley, Conrad and Thomson's letter frequency count. The columns 


\section{TABLE 3}

Relation between observed and expected error frequencies in columns of the keyboard.

\section{Keyboard Columns}

$\begin{array}{lcccccccccc} & \text { qaz } & \text { wsx } & \text { edc } & \text { rfv } & \text { tgb } & \text { yhn } & \text { ujm } & \text { ik } & \text { ol } & \text { p } \\ \text { EXPECTED } & 9.5 & 6.9 & 15.9 & 9.5 & 13.7 & 13.4 & 6.2 & 9.6 & 12.8 & 2.5\end{array}$

\section{HoRIZONTAL}

\begin{tabular}{llll:r|r|rrrrr} 
Missed & 1.4 & 8.8 & 14.8 & 26.4 & 23.0 & 4.7 & 7.5 & 6.1 & 4.1 & 0.7 \\
Typed & 2.0 & 8.1 & 13.1 & 24.6 & 21.6 & 15.8 & 6.8 & 5.4 & 4.1 & 0.0
\end{tabular}

VerTical

\begin{tabular}{|c|c|c|c|c|c|c|c|c|c|}
\hline Missed & 10.2 & 24.5 & 32.7 & 12.2 & 6.1 & 6.1 & 0.0 & 2.0 & 6.1 \\
\hline Typed & 8.9 & 26.7 & 28.9 & 13.3 & 6.7 & 6.7 & 0.0 & 2.2 & 6.7 \\
\hline
\end{tabular}

Equivocal

Postponed

$\begin{array}{lll}9.1 & 8.0 & 15.8\end{array}$

$12.4 \quad 10.2$

9.1

$9.1 \quad 13.6$

2.3

Advanced

$\begin{array}{lll}5.7 & 3.4 & 26.1\end{array}$

6.8

$6.7 \quad 11.4$

$11.4 \quad 17.1$

4.5

containing " $\mathrm{r}, \mathrm{f}, \mathrm{v}$ " and " $\mathrm{t}, \mathrm{g}, \mathrm{b}$ " show horizontal etrors, both missed and typed, which are considerably in excess of expectation. The missed letter was the one which should have been typed, and the typed letter was the one typed in error. (Note that both columns are typed by the left index finger.) Vertical errors show the same trend for two other left hand columns; those containing "w, s, x" and " $e, d, c$ ". Both these trends are encased in dotted lines in the table. Equivocal errors show column frequencies close to those expected. Reversals and omissions were too few in number to make this type of analysis profitable.

The relation between observed and expected error percentages for rows of the keyboard is shown in Table 4. Horizontal errors, both missed and typed, tend to occur more than expected in the bottom row and less in the top row. There are two main trends for vertical errors. One trend is for fewer errors than expected to be made on the top row. The other is for letters to be missed more than expected in the bottom row, and typed more than expected in the home row. This latter trend is seen encased in solid lines in the table. Equivocal errors show a slight tendency for advanced letters to occur more than expected in the top row and less than expected in the bome row.

Letter frequency. The 26 letters of the alphabet were arranged in decreasing order of expected frequency, as derived from the Baddeley, Conrad and Thomson data, 


\section{TABLE 4}

Relation between observed and expected error frequencies in rows of the keyboard.

\begin{tabular}{|c|c|c|c|}
\hline & $T o p(1)$ & Home (2) & Bottom (3) \\
\hline EXPECTED & 52.8 & 30.4 & 16.8 \\
\hline \multicolumn{4}{|l|}{ HoRIzONTAL } \\
\hline Missed & 46.9 & 29.4 & 24.2 \\
\hline Typed & 46.1 & 28.3 & 25.1 \\
\hline \multicolumn{4}{|l|}{ Vertical } \\
\hline Missed & 32.7 & 32.7 & 34.7 \\
\hline Typed & 27.7 & $57 . \overline{4}$ & 14.9 \\
\hline \multicolumn{4}{|l|}{ EQUIVOCAL } \\
\hline Postponed & 51.1 & 29.5 & 19.3 \\
\hline Advanced & 63.6 & 23.9 & 11.3 \\
\hline
\end{tabular}

and then split into 3 groups of approximately equal total frequency, as a basis for a $\chi^{2}$ analysis of the obtained error data. These groups were : " $e, t, a$ " $-29.8 \%$, " $\mathrm{i}, \mathrm{o}, \mathrm{h}, \mathrm{n}, \mathrm{r}$ "- $36.9 \%$ and all remaining letters $33.3 \%$. The relation between letter frequencies of the two largest categories of error, horizontal and equivocal, and these expected frequencies, is shown in Table 5. Horizontal errors, both missed and typed, occurred on infrequent letters (i.e., the " remaining letters" group) significantly beyond chance. Equivocal errors for both advanced and postponed letters were quite close to expected values. It should be noted that there is probably a "built-in" tendency to type infrequent letters in horizontal spatial errors, since the highly frequent letters, at which most strokes are directed, are surrounded by letters of lesser frequency. Nonetheless, missed letters in horizontal errors, which are not subject to any such bias, show a very similar frequency distribution to typed letters.

Digram frequency. Digrams formed by the error and letter which preceded it were compared in frequency with the correct digrams which would otherwise have occurred. But such a comparison is complicated by the fact that, by chance, erroneous digrams can include many digrams which do not occur in the language. (For instance in the Baddeley et al. count, $41.6 \%$ of possible digrams do not occur.) Therefore, unless there was a tremendous tendency for errors to form digrams of high frequency in the language, the error digrams would be considerably lower in mean frequency than the correct digrams they replaced. This was what the analysis showed. 


\section{TABle 5}

The relation between observed and expected frequencies of letters in horizontal and equivocal errors.

\section{HORIZONTAL}

\section{Frequency}

group

$\begin{array}{lcccccccc} & \text { Obs. } & \text { Ex. } & \text { Obs. } & \text { Ex. } & \text { Obs. } & \text { Ex. } & \text { Obs. } & \text { Ex. } \\ \text { e, t, a, } & 26 & 43.8 & 28 & 44.1 & 25 & 25.9 & 27 & 26.2 \\ \text { i, o, h, n, r } & 34 & 54.3 & 45 & 54.6 & 28 & 32.2 & 30 & 32.5 \\ \text { Other } & 87 & 49.0 & 75 & 49.3 & 34 & 28.9 & 31 & 29.2 \\ & & & & & & & & \\ & & & & & & & & \end{array}$

(Typed and advanced columns total one less than missed and postponed columns respectively, because one error in the former two categories involved punctuation.)

A result not biased by non-occurrence contingencies can be gained by comparing the frequencies of the correct versions of digtams on which errors occurred with the frequencies of those digrams in the language. This was done by ranking the expected digrams according to their frequency in the Baddeley, Conrad and Thomson count, and then splitting the digrams up into 5 roughly equal frequency groups in a manner similar to that described in the analysis of letter frequency. $\chi^{2}$ tests showed that both horizontal and equivocal errors showed a significant tendency to occur on less frequent digrams.

Syllable variobles. Any direct assessment of the relation of errors to syllables is hindered by the problem of defining the syllabls. Syllabification of many words differs depending on whether speech or orthography is being considered. Although orthographic considerations are probably more important to a typist, if typists are influenced by syllabic factors at all, it is not always clear where orthographic syllable boundaries lie. However, an attempt was made to divide polysyllabic words containing errors into syllables from the orthographic point of view, making arbitrary decisions where the position of the boundary was not intuitively clear. As a basis for analysis, the assumption was made that if errors occurred at random throughout the words, the proportion of errors falling on syllable boundaries would be similar to the proportion of syllable boundaries to all letter boundaries in the corpus (not counting the word boundary as a syllable boundary). $\chi^{2}$ tests showed this assumption to be upheld in all cases but one : horizontal errors occurred significantly less than expected immediately before syllable boundaries. 

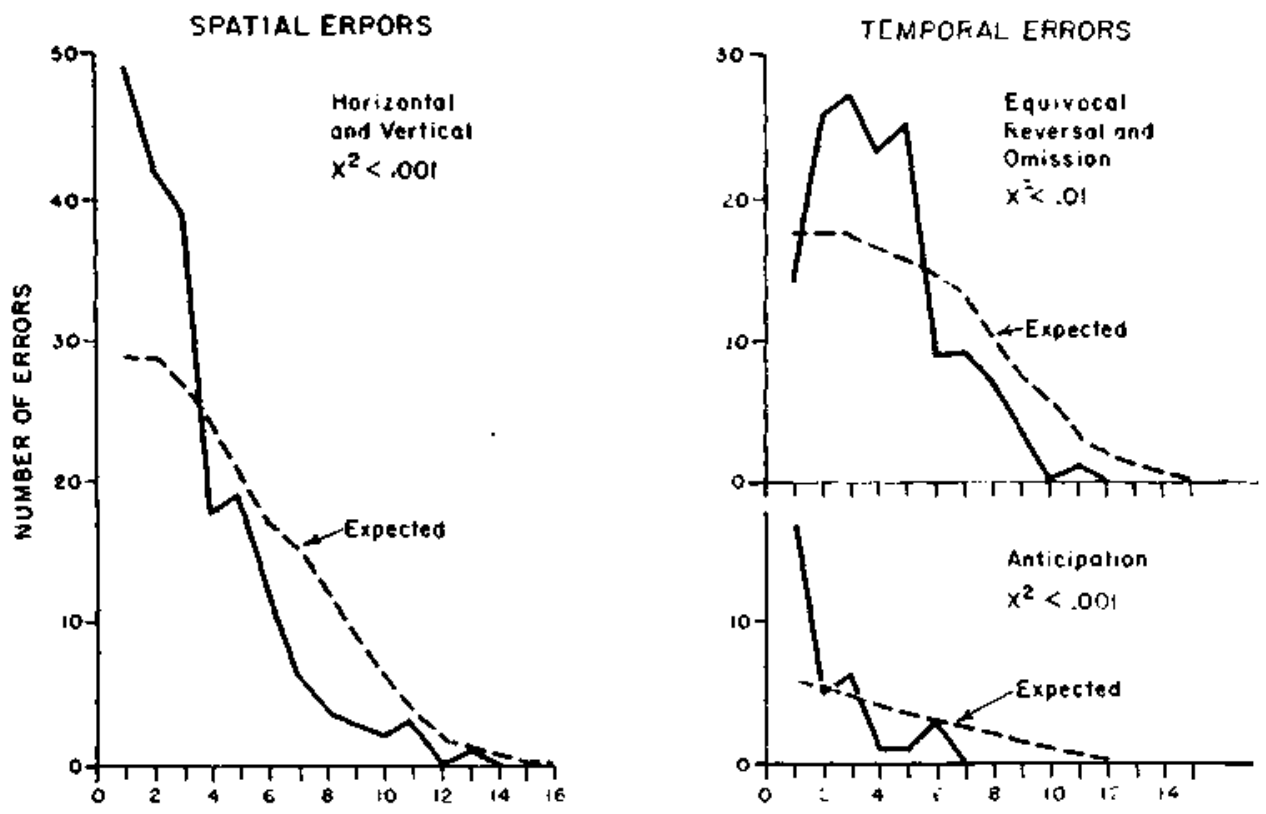

LETTER POSITION

Fig. 2. The relation between obtained (solid lines) and expected (dotted lines) error frequencies for spatial and temporal errors as a function of the position of the letter in the word.

Position of error in the word. Fig. 2 shows the number of temporal and spatial errors occurring in each letter position. The dotted lines represent the expected error frequency in each letter position. This was derived from the proportion of letters in each position to the total number of letters in the words containing the etrors. The 2 spatial error categories, and the equivocal, reversal, and omission categories wete combined, as they showed similar trends. $\chi^{2}$ tests showed that obtained error frequencies departed significantly from expected frequencies in all 3 cases. All distributions show a less than chance frequency of errors in the later parts of words. Spatial errors occur considerably more often than expected on the first 3 letters. More than one half of all anticipations occur in the initial position. Inspection of the data shows that approximately $80 \%$ of these errors were either first letters of the next word or first letters of the next syllable. The three other types of temporal error occur more than expected on early letters of words, except for the initial position, where they are less frequent than expected. This trend occurred in all three error categories taken individually. 
TABLE 6

Average lengths of words containing the various types of error.

$\begin{array}{cc}\text { Spatial. } & \text { Letters } \\ \text { Horizontal } & 6.8 \\ \text { Vertical } & 7.3 \\ & \\ \text { TEMPoral } & \\ \text { Equivocal } & 7.8 \\ \text { Reversal } & 8.4 \\ \text { Omission } & 9.6 \\ \text { Anticipation } & 6.0\end{array}$

Word size. Average lengths of the words containing errors are shown for each of the sub-categories of spatial and temporal errors in Table 6. A non-parametric one-way analysis of variance (Kruskal-Wallis, 1952) of all categoties except anticipation was significant beyond the 0.001 level. A median test between all spatial errors and all temporal errors then showed that spatial errors occur on shorter words than do temporal errors with a significance beyond the 0.01 level. (Anticipation errors were not included, as their average word length was probably influenced by the classifying procedure. A letter was less likely to be classified as an anticipation etror, the earlier the letter was in a word in relation to the one it could have been anticipating. This perhaps artificially reduced the size of words in which anticipation errors were judged to occur.)

Letter context. An analysis was made of the relation between errors and letters which occurred twice in a word. The assumption was made that if errors occurred at random with respect to doubly occurring letters, the proportion of errors involving doubly occurring letters would be similar to the proportion of doubly occurring letters to all letters. $\chi^{2}$ tests showed that the assumption was not upbeld in any of the temporal error categories, but was upheld in all but one of the spatial categories. In temporal errors (i.e., equivocal, reversal and omission errors), both advanced and postponed strokes were always significantly associated with letters occurring twice in the word. (Anticipation errors could not be analysed because they sometimes stretched across word boundaries.) In spatial errors, the only significant tendency was for letters missed in spatial errors to be less associated with doublets than expected. This result may be due to the tendency, observed earlier, for spatial errors to occur on infrequent letters. When the same letter as the error letter was found elsewhere in the word it occurred about equally often before and after the error. 
Miscellaneous. There was no obvious variable associated with the insertion of the particular letters found in the 11 interpolation errors. Phonemic errors occurred so infrequently (2) as to make their existence as a distinct phenomenon doubtful. Contralateral errors occurred only 11 times. Nevertheless, they are probably due to a specific malfunction rather than random variability. If 11 of these could occur at random, the "unclassifiable" total in this study would probably be much higher than 69 . Dynamics errors were few (7) but again, they are not easily explained as random effects. Type errors were of two distinct kinds : changes in suffix, occurring in 9 words with an average word length of $\mathbf{8 . 3}$ letters, and production of an entirely different word from that intended, occurring in 13 words with an extremely short average word length of 3.4 letters. These words were all very common, e.g., the, that, than, of, on.

\section{Discussion}

Spatial errors were found, in general, to occur significantly more often on the left hand. But analysis of keyboard variables showed that these errors were not evenly spread over the left hand keyboard, which would suggest that they were simply due to the well-known lesser dexterity of the left hand. The results, however, are consistent with the possibility that, despite the mixed handedness of 2 of the subjects, the group in general is less dexterous with the left hand. However, this results in errors only under conditions of particular difficulty : i.e., when a downward movement of inside fingers of the hand is required as in the case of vertical errors, and when a single finger has 1 of 2 spatially similar alternatives to type, as in the case of index finger involvement in horizontal errors. The possibility that the difficulty may be one of movement in vertical errors, and one of "decision" between alternatives in index finger action is suggested by one particular detail of the results. As Table 4 shows, vertical errors consisted mostly in missing the stroke requiring most movement (bottom row) and typing the stroke requiring least movement (bome row). On the other hand, as Table 3 shows, index finger errors were made as often by typing any given letter as by missing it, suggesting a confusion of adjacent possibilities. In contrast to spatial errors, temporal errors were made about equally often with each band, and they showed no marked tendency to be influenced by the structure of the keyboard.

Temporal and spatial errors also showed contrasting relations to letter frequencies in the language, when horizontal spatial, and equivocal ertors were taken as representative of spatial and temporal errors respectively. In equivocal errors both the anticipated and postponed letters were so highly related to letter frequency as to suggest merely that the more frequently a letter occurred the more likely it was to be involved in an equivocal error. This is much what one would expect if letter frequency had merely a chance relation to temporal errors. On the other hand, horizontal spatial errors, both typed and missed strokes, occurred significantly more often on the least frequent letters in the language. The result for typed strokes is 
probably an artifact, as explained earlier. The result for missed strokes may be partly due to subjects' having less practice at making less frequent strokes, but it must also be partly due to the fact that less frequent letters are often in awkward positions on the keyboard. To the extent that this is an influence, the present results support the earlier conclusion that spatial errors are affected by keyboard difficulty variables while temporal errors are not.

When digrams, the second letter of which was an error, were examined, both borizontal and equivocal errors tended to occur largely on less frequent digrams in the language. For horizontal errors, this result is correlated with the one just described for single letter frequency, as less frequent letters tend to result in less frequent digrams. Therefore, the relative importance of the digram sequence itself cannot readily be assessed. But in equivocal errors, where less frequent letters do not predominate, the present result suggests that infrequent sequences themselves provide some special difficulty for the mechanism controlling sequences.

Digram frequency is thus the first variable mentioned that is of importance in temporal errors. Handedness, keyboard structure and letter frequency are not. A second variable found to be important in temporal errors is word length. Temporal errors tend to occur on longer words than spatial errors, and it can be argued that the more serious the temporal ertor, the longer the word in which it usually occurs. Of the three sub-types equivocal, reversal, and omission, equivocal errors, which are discovered immediately, occur, on the average, in the shortest words. Reversal errors, in which all correct letters are typed, but where two letter positions are swapped without immediate discovery, occur on the next shortest length of word. The longest words involve leaving out a letter altogether, and not noticing the omission at the time. Unfortunately, it cannot be decided in this study whether word length itself is the most important variable here, because it is well known that, in general, the longer the word, the less frequently it occurs in the language (Zipf, 1935) and this fact is probably of some importance in typing.

The word boundary influences the occurrence of ail spatial and temporal error categories. The high frequency of anticipation errors immediately following the word boundary and the high frequency with which these errors consisted of initial letters of the next word or syllable, suggests that at the word boundary a decision is made as to which word or syllable, not merely which letter, is to occur next. The possibility that a unit larger than the letter is involved is strengthened by the infrequent occurrence of equivocal, reversal, and omission errors on the first letter of words compared with their frequencies of occurrence on $2 \mathrm{nd}, 3 \mathrm{rd}$ and 4 th letters. If decisions were being made letter by letter, one would expect the second letter of a word to be advanced to first position much more often. This apparent temporal certainty about the position of the first letter in the word may result in "commands" at this stage sometimes being issued too quickly for the motor system to deal with, thus producing the observed high frequencies of spatial errors on letters early in words. A study of the timing or the amplitude of typed strokes in relation to word boundaries could provide information on this possibility. 
An effect of word structure on the sequencing process was shown up in the analysis of the influence of doubly occurring letters on temporal errors. Letters which occurred twice in a word were about equally likely to result in advancing and postponing one of such letters in the sequence. The effect seems therefore, to be one of confusing the sequence rather than largely facilitating or largely inhibiting any aspect of it,

Some of the miscellaneous errors, although infrequent in occurrence, seem to be of considerable theoretical importance. Type errors tesulting in a different bighly frequent word, or a different suffix, are of interest as they appear to involve error at the "semantic level" of the output mechanism, or at the level of word choice (see Osgood, 1963, for a language producing schema with a semantic stage). Dynamics errors are of importance for theories of serial order in general, as Lashley (1951) bas pointed out, because they suggest that the mechanism determining the serial activation of response units is relatively independent of the response units themselves. Contralateral errors are of interest because the letter typed involves analogous anatomical apparatus to the one which should have been typed, and that fact appears to potentiate the contralateral action.

A hundred or so multiple classification errors have not been analysed because their relatively low frequencies and the ambiguity of their status appeared to make analysis unprofitable. But this does not mean that they are unimportant indices of the mechanism of typing, because variables responsible for more than one type of error may combine to produce many of these errors, as their classification suggests. Such multiple determination of errors may be of especial importance in perseverative errors. This is a type of error, not separated out by the present error classification scheme, where typists persist in making a particular kind of error. Favourite ones in the present sample included beginning "conditioning" with a " $\mathrm{d}$ " (vertical and anticipation error) and beginning "reinforcement" with a "ri-" (contralateral and equivocal error). Such errors require a distinction between a response mechanism which errs temporarily, and a "determining tendency" or "idea" which remains the same, as Lashley (1951) has suggested.

The most general result of the present study has been the extent to which separate variables have been associated with spatial and temporal errors. The result suggests a distinction between an "executive" mechanism reflected by spatial errors, and a "programming" mechanistn reflected by temporal errors. The executive mechanism is particularly distinguisbed by its sensitivity to the physical difficulty of the behaviour whetber produced by limitations of the non-preferred hand, difficulties of making particular finger movements and perhaps particular sequences of movement, or difficulty in executing commands early in words. The "programming" mechanism is distinguished by its sensitivity to certain variables of language structure such as word boundaries, word length, double occurrence of a letter in a word, and infrequent letter sequences. The programming mechanism seems to act largely as an open loop system in that there is only one piece of evidence that the feedback accompanying difficulties of the executive mechanism might influence its output, i.e. the effect of infrequent digrams which also trouble the executive mechanism. 
What are the implications of the present results for existing theories of serial ordering ? Theories that explain serial ordering as "chaining" by postulating that each response produces the stimulus for the next response (Skinner, 1958, Keller and Schoenfeld, 1950) cannot account for many aspects of the present results. In general, these types of "single stage" theories, as Osgood (1963) called them, are not consistent with the apparent existence of the two types of mechanism, "programming " and "executive", that the present results require. Further, as mentioned previously, perseverative errors require an additional distinction between a "derermining tendency" which remains unchanged, and a response-producing apparatus which errs. This is also not consistent with any single stage theory. In addition there are many types of error in the present data which are not accountable for in terms of antecedent variables favoured by the above theorists, such as response strength as a function of frequency and/or amount of reinforcement, presence of particular discriminative stimuli in the experimental situation, and recency of reinforcement, even if such variables could be specified. For instance, in temporal errors, which are the category most relevant to chaining theory, there was no beyond-chance tendency to advance more frequent letters in the sequence. In fact, letters were postponed as well as advanced, independently of frequency. Similarly, anticipation errors did not take the form of typing more frequently occurring letters. Reversal errors were contrary to chaining theory for another reason; the advanced letter should have provided a stimulus for the letter which usually followed it, but it was the letter which usually preceded it that was typed next. Now chaining theorists do not necessarily consider the letter to be the functional unit in the chaining process, but if they choose a larger category they are then unable to explain single letter errors such as omissions and contralateral errors which are preceded and followed by the correct letters.

The results are more consistent with the notion that there is a hierarchy of determinants of output as Lashley (1951), Miller, Galanter and Pribram (1960), and Osgood (1963) have suggested. Osgood, for example, has suggested a three stage production mechanism for speech : (1) intention (or meaningful level), (2) skill (or integrational level) and (3) execution (or projection level). This classification is analogous to the one which appears appropriate to the present data: (1) determining tendency (after Lashley), (2) programming mechanism and (3) executive mechanism. To account for the second stage of production to which most of the present results are relevant, Osgood has suggested an integration principle derived from Hebb's theorizing (Hebb, 1949) :- The greater the frequency with which stimulus events (S-S) or response events (R-R) have been paired in the input and output experience of the organism, the greater will be the tendency for their central correlates to activate each other. But although frequency of letter sequences has had some rôle in influencing integration in the present study, much more influence can be attributed to a number of properties of the programming mechanism itself in determining what will remain as integrated sequences and what will fragment during typing. What are these properties? Many pieces of evidence point to a programmer which is activating, to varying degrees, a number of units stretching some distance ahead of the current response in time. 
(Note that Ruch (1951) has suggested a possible neurological basis for such a system in the form of a cerebral-cerebellar circuit "by which an instantaneous order can be extended forward in time. Such a circuit, though uninformed as to the consequences, could, so to speak, "rough in' a movement _._.".) Temporal errors on letters occurring twice in a word especially suggest such a mechanism; one which is "confused" by the existence of two identical commands at about the same time in the programme and manifests this confusion by displacing one of the commands. It may be of some value to think of the size of the functional unit of this programming mechanism as varying sensitively with the results of its previous actions, and the structure of the following ones. Part of such variation is illustrated by the tendency for the functional unit following the word boundary to be of syllabic or perhaps sometimes word size. But on longer words, the functional unit probably decreases in size, as suggested by the tendency of these wotds to be fragmented by temporal errors.

\section{REFERENCES}

Baddeley, A. D., Conrad, R. and Thomson, W. E. (1960). Letter structure of the English. language. Nature, 186, $4722,414$.

Dvorak, A., Merricx, N. L., Dealey, W. L. and Ford, G. C. (1936). Typewriting Behavior (New York).

HeBB, D. O. (1949). The Organization of Behavior (New York).

Keller, F. S. and Schoenfeld, W. N. (1950). Principles of Psycho'ogy (New York).

KruSkal, W. H. and Wht.tis, W. A. (1952). Use of ranks in one-criterion variance analysis. Jour. Amer. Srat. Ass., 47, 583.

LASHLEY, K. S. (1951). The problem of serial order in behavior. In L. A. Jeffress (ed.), Cerebral Mechanisms in Behavior (New York).

Mirler, G. A., Galanter, E. and Pribram, K. H. (1960). Plans and the Structure of Behavior (New York).

OsGoon, C. E. (1963). On understanding and creating sentences. Amer. Psychol., 18, 735.

RUCH, T. C. (1951). Motor systems. In S. S. Stevens (ed.), Handbook of Experimental Psychology (New York).

Skinner, B. F. (1958). Verbal Behavior (New York).

ZIPF, G. K. (1935). The Psychology of Language (Boston). 
Copyright of Language \& Speech is the property of Kingston Press Ltd. and its content may not be copied or emailed to multiple sites or posted to a listserv without the copyright holder's express written permission. However, users may print, download, or email articles for individual use. 